\title{
Feature Extraction for the Prognosis of Electromechanical Faults in Electrical Machines through the DWT
}

\author{
J.A. Antonino-Daviu, M. Riera-Guasp, M. Pineda-Sánchez, J. Pons-Llinares, R. Puche-Panadero, J. Pérez-Cruz \\ Instituto de Ingeniería Energética, Universidad Politécnica de Valencia, Camino de Vera s/n \\ 46022 Valencia, SPAIN \\ E-mail: joanda@die.upv.es \\ www.upv.es \\ Received: 29/09/08 Revised: 19/05/09
}

\begin{abstract}
Recognition of characteristic patterns is proposed in this paper in order to diagnose the presence of electromechanical faults in induction electrical machines. Two common faults are considered; broken rotor bars and mixed eccentricities. The presence of these faults leads to the appearance of frequency components following a very characteristic evolution during the startup transient. The identification and extraction of these characteristic patterns through the Discrete Wavelet Transform (DWT) have been proven to be a reliable methodology for diagnosing the presence of these faults, showing certain advantages in comparison with the classical FFT analysis of the steady-state current. In the paper, a compilation of healthy and faulty cases are presented; they confirm the validity of the approach for the correct diagnosis of a wide range of electromechanical faults.
\end{abstract}

Keywords: electric machines, fault diagnosis, wavelet transform, broken bars, eccentricities.

\section{Introduction}

Electrical induction machines (also known as asynchronous machines) are deeply spread in many industrial applications. Unforeseen faults in these machines might lead to high costs, since they are often critical elements in those processes in which they operate. Due to this, the diagnosis of the possible faults occurring in these devices has become a topic of special interest and concern in the industrial environment [1] within the context of current concern on diagnosis issues [2]. The development and optimization of diagnosis techniques being able to detect the possible failures in an earlier stage have been the motivation of many works in the literature during these last few years. Studies on the occurrence of electromechanical faults in asynchronous machines [1] show a significant percentage of faulty events related to the rotor, such as rotor asymmetries (rotor bar breakages, cracked end rings...) and various modalities of eccentricities (static, dynamic or mixed eccentricities); they have been deeply analyzed in the literature due to their particular hazard caused by the progressive propagation or the possibility of rotor to stator rub [3-5].

These faults cause some effects in the different electromechanical quantities of the machine (currents, vibrations, fluxes, torque...) which may help to diagnose the presence of the corresponding failure. In fact, some studies have investigated the effect that each particular fault provokes on the different electrical quantities, trying to obtain the most suitable for diagnosing the presence of each failure, according to its sensitivity, non-invasive nature and other criteria.

The most common approach for the diagnosis of most of the faults (for instance, rotor asymmetries, different types of eccentricities or inter-turn short-circuits) in the industrial environment is based on the analysis of the current demanded by the machine; this quantity can be measured in a non-invasive way, this is, without interference on the usual operation of the machine. Moreover, the equipment required for capturing the current signal is very simple and also the software needed for its computation.

The traditional diagnosis method is focused on applying the Fourier transform to the current demanded by the 
machine during its steady-state operation. Under ideal operation and healthy condition, this should be a pure sinusoidal signal, so the Fourier analysis would reveal the presence of a single frequency component at the supply frequency. Nevertheless, even under healthy condition, this spectrum is usually polluted by other frequencies caused by the slotting, non-ideal winding distribution, perturbations in the operation of the machine, noises, transient oscillations or even rotor imperfections due to the manufacturing process [6-7].

In the case of a machine with rotor asymmetries or a machine with certain level of eccentricity, some particular frequency components appear in the Fourier spectrum of the steady-state current. Many authors have studied the frequencies amplified by the presence of these faults; these works have led to expressions that have become very common in the industrial environment for diagnosis purposes; for instance, in the case of rotor bar breakages, the main frequencies amplified by the presence of the fault are given by (1) (where $s$ is the slip $\left(s=n_{s}-n / n_{s}\left(n_{s}=\right.\right.$ synchronous speed and $n=$ speed) and $f$ is the supply frequency ( $50 \mathrm{~Hz}$ in Europe)) and they are known as sideband components [4]. These components are shown in Figure 1, corresponding to a loaded machine with two broken bars. Analogue expressions are obtained for the case of static, dynamic or mixed eccentricities.

$$
f_{S C}=f \cdot(1 \pm 2 \cdot s)
$$

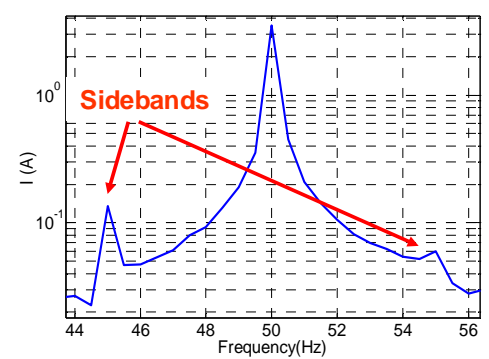

Fig. 1. FFT of the steady state current for a loaded machine with two broken bars.

This classical approach based on the steady-state analysis of the current, has some drawbacks reported by several authors [6-7]; for instance, when the machine is unloaded or lightly loaded, the diagnosis of rotor asymmetries or even eccentricities can become specially difficult due to the low value of the slip [6-7], causing that the frequency components used for the diagnosis overlap the frequency of supply ( $f$ ) (Figure 2(a)). Moreover, other common phenomena such as load fluctuations or voltage oscillations can introduce frequencies very close to those amplified by the previous faults, leading to confusion or even to a wrong diagnosis (Figure 2(b)).

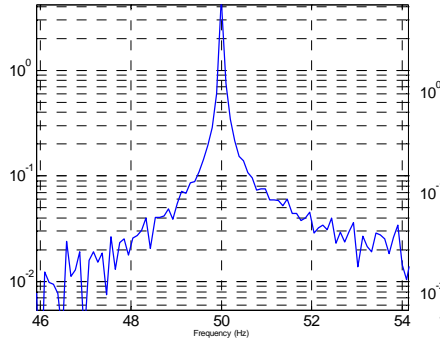

(a)

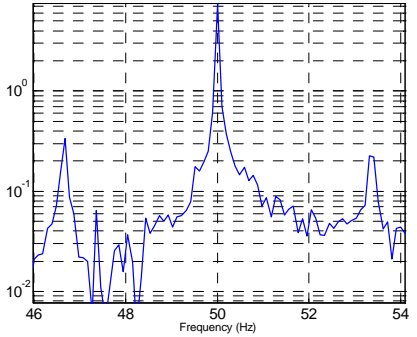

(b)
Fig. 2. FFT of the steady state current for: (a) unloaded machine with two broken bars (b) healthy machine with fluctuating load torque.

Due to all these facts, some authors have proposed the study of the transient processes of the machine as an alternative way to obtain additional information which could complement that provided by the steady-state methods. In this context, the study of the current during the connection process of the machine (startup transient) has drawn most of the attention [7-12]. The implicit common basis of these methods is the detection of the evolution during that transient of certain characteristic components created by the corresponding fault.

In this context, a new methodology based on the application of the Discrete Wavelet Transform (DWT) to the startup current, and the subsequent study of the wavelet signals resulting from the transform was proposed recently [7-12]; these signals enable not only the mere detection, but also the extraction of the evolution during the transient of the components created by each fault, arising characteristic patterns that could be used for the reliable diagnosis of the fault. The further automatic recognition of these patterns, using modern image recognition algorithms would enable the on-line diagnosis of the corresponding fault as well as the quantification of the degree of severity.

The aim of this paper is to review the proposed diagnosis methodology, presenting a compilation of different cases. These experimental cases are referred to 
a $1.1 \mathrm{~kW}$ machine operating under various conditions and with different faults. In some of the presented cases, the classical diagnosis method, currently used in the industrial environment and based on the application of the FFT to the steady-state current, is not suitable or leads to a confusing diagnostic. Moreover, computational aspects of the diagnosis algorithm are analyzed, emphasizing the issues for its practical implementation. The results show the validity of the method for a reliable fault diagnosis. This might lead to the possible future implementation of portable condition monitoring devices based on this methodology.

\section{Electromechanical faults during the startup}

Two main faults are considered in the paper; broken rotor bars and mixed eccentricities (combination of static and dynamic eccentricities).

\subsection{Broken rotor bars}

The presence of broken rotor bars introduces, in the steady-state current spectrum, two sideband components around the supply frequency $(f=50 \mathrm{~Hz})$, with frequencies given by (1). During the startup transient, the slip $s$ changes from 1 to a value very close to 0 . As the slip $s$ varies, the frequency of the component with negative sign in (1) (left sideband component) also changes; it decreases first from a value equal to the supply frequency $f$ to $0 \mathrm{~Hz}$ and it increases again up to reaching a value very close to the supply frequency $f$ [7]. Its amplitude also evolves in a very characteristic way [8]. The extraction of that characteristic transient waveform has revealed as a reliable way for diagnosing the presence of the asymmetry in the machine.

\subsection{Mixed eccentricities}

Some authors [3] have deduced a general expression for the frequencies amplified by mixed eccentricities:

$$
f_{e c c}=f \cdot\left[\left(1 \pm m \cdot\left(\frac{1-s}{p}\right)\right)\right]
$$

where $p=$ number of pole pairs and $m=1,2,3 \ldots$

As it was proven in previous works [9], the slip variation during the startup leads to a particular evolution of the frequency components created by the eccentricity. For $m=p / 2$, considering $f=50 \mathrm{~Hz}$, two frequency components with very characteristic evolutions appear; one of them evolving during the transient from $50 \mathrm{~Hz}$ to almost $25 \mathrm{~Hz}$ and the second changing from $50 \mathrm{~Hz}$ to around $75 \mathrm{~Hz}$ [9]. This variation, totally different from that for the broken bars, can be also used for the diagnosis of the eccentricity.

\section{Discrete Wavelet Transform (DWT)}

The main idea that underlies the application of the DWT is the dyadic band pass filtering process carried out by this transform. Provided a certain sampled signal $\mathrm{s}=\left(\mathrm{i}_{1}\right.$, $\left.\mathrm{i}_{2}, \ldots \mathrm{i}_{\mathrm{N}}\right)$, the DWT decomposes it onto several wavelet signals (an approximation signal $a_{n}$ and $n$ detail signals $\left.d_{j}\right)[7,13]$. A certain frequency band is covered by each wavelet signal; the wavelet signal reflects the time evolution of the frequency components of the original signal s which are contained within its associated frequency band $[7,14]$.

More concretely, if $f_{s}$ (samples/s) is the sampling rate used for capturing $\mathrm{s}$, then the detail signal $d_{j}$ contains the information concerning the signal components with frequencies included in the interval:

$$
f\left(d_{j}\right) \in\left[2^{-(j+1)} \cdot f_{s}, 2^{-j} \cdot f_{s}\right] \mathrm{Hz}
$$

The approximation signal $a_{n}$ covers the low frequency components of the signal, belonging to the interval:

$$
f\left(a_{n}\right) \in\left[0,2^{-(n+1)} \cdot f_{s}\right] \mathrm{Hz}
$$

Therefore, the DWT carries out the filtering process shown in Figure 3. Note that the filtering is not ideal, a fact leading to a certain overlap between adjacent frequency bands $[7,12,15]$. This causes some distortion if a certain frequency component of the signal is close to the limit of a band.

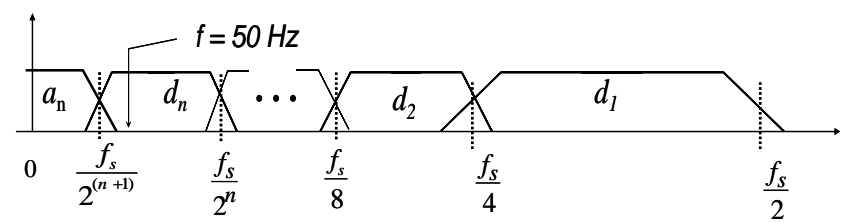

Fig. 3. Filtering process performed by the DWT.

Due to the automatic filtering performed by the wavelet transform, the tool provides a very attractive flexibility for the simultaneous analysis of the transient evolution 
of rather different frequency components present in the same signal. At the same time, in comparison with other tools, the computational requirements are low. In addition, the DWT is available in standard commercial software packages.

\section{Practical algorithm for the diagnosis methodology}

Let $i(t)$ denote the startup current signal, i.e, the current demanded by the machine during the connection process (startup transient). In the case of healthy machine, this is a $50 \mathrm{~Hz}$ (supply frequency) sinusoidal signal whose amplitude decreases progressively from an initial value towards the steady-state value. This signal is given by (5), where $\hat{I}(t)$ is the RMS value of the current.

$$
i(t)=\sqrt{2} \cdot \hat{I}(t) \cdot \operatorname{sen} 2 \cdot \pi \cdot 50 \cdot t
$$

In the case of a faulty machine, fault-related frequency changing components coexist during the transient, together with the fundamental current component $i(t)$. Usually, they have much lower amplitude in comparison with $i(t)$. This is the case of broken rotor bars. When this fault is present, several components appear, with characteristic variations in frequency and amplitude during the startup. The most relevant used for the breakage diagnosis is the Left Sideband Component (LSC), whose frequency is given by (6).

$$
f_{L S C}=f \cdot(1-2 \cdot s)
$$

As justified in previous works [8], the theoretical waveform of this component during the transient is according to that shown in Figure 4. As observed, the frequency evolution of this component is in agreement with that described before; first, it decreases from the supply frequency $(f=50 \mathrm{~Hz})$ to $0 \mathrm{~Hz}$ and later it increases towards the steady-state value (near $50 \mathrm{~Hz}$ ). Therefore, the frequency evolution of the LSC is constricted to a certain frequency band $([0-50] \mathrm{Hz})$. The amplitude of the LSC has also a characteristic evolution, being always much lower than $i(t)$.

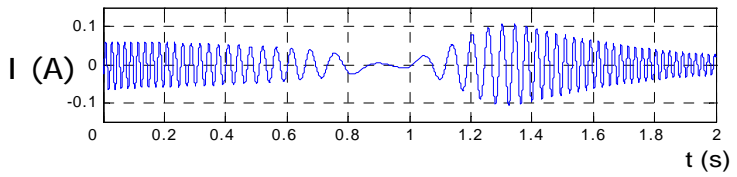

Fig. 4. Transient evolution of the LSC during the startup.
In the case of mixed eccentricities, two relevant faultrelated components coexist with the fundamental component $i(t)$. In this case, their frequency evolutions are rather different, as commented in Section 2.2, but they have also lower amplitude than $i(t)$.

The objective of the DWT-based algorithm is to perform a band pass filtering of the startup current signal $\left(i_{s t}(t)=i(t)+\right.$ fault-related components $\left.)\right)$. In this way, since the signal $i(t)$ is a pure $50 \mathrm{~Hz}$ component, whereas the fault-related components evolve in different frequency bands, the DWT enables their separation, being able to diagnose the presence of the corresponding fault. Moreover, due to the timefrequency decomposition carried out by the DWT, characteristic patterns appear in the time-frequency plane, which are caused by the evolution of fault-related components. These patterns are different for each type of fault, thus becoming reliable indicators of the presence of the corresponding fault.

According to these comments, the complete sequence of steps for the practical application of the diagnosis algorithm is shown in Figure 5.

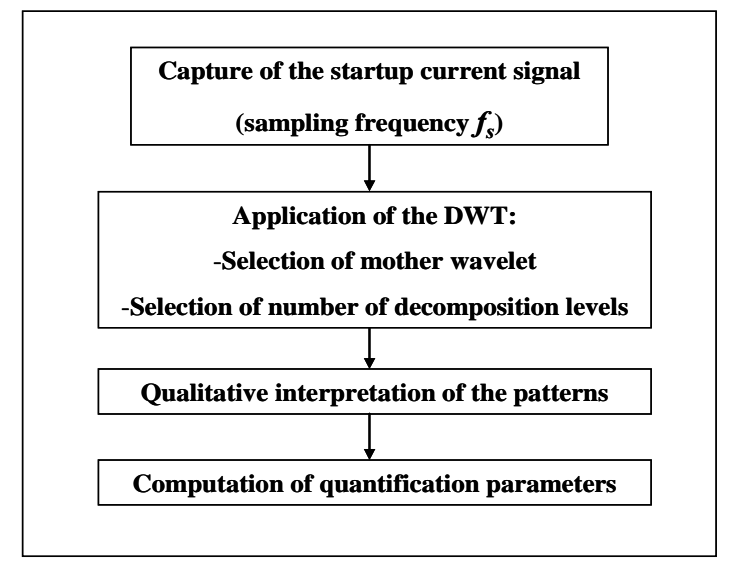

Fig. 5. Scheme for the diagnosis algorithm.

\section{Capture of the startup current signal}

The current demanded by the machine during the startup transient $i_{s t}(t)$ can be easily captured with very simple equipment such a Hall sensor or a current probe. In addition to the simplicity of the required equipment, the capture of this quantity can be carried out in a noninvasive way, this is, without interfering the normal operation of the machine. This is a crucial advantage for many industrial processes, whose interruption might lead to disastrous consequences. For the application of the methodology, taking into account the Mallat 
algorithm and the Nyquist criterion, a sampling frequency $f_{s}$ around 5000 samples/second is enough, as justified in previous works [12].

Regarding the length of the current register, it should cover the whole duration of the startup. This depends on the motor size, the inertia or the motor-load group and other parameters.

\section{Application of the DWT}

The next step consists of the application of the DWT to the captured current signal $i_{s t}(t)$. The mathematical expression for the one-dimensional DWT decomposition of the signal $i_{s t}(t)$ is given by (7).

$$
i_{s t}(t)=\sum_{i} \alpha_{i}^{n} \cdot \varphi_{i}^{n}(t)+\sum_{j=1}^{n} \sum_{i} \beta_{i}^{j} \cdot \psi_{i}^{j}(t)=a_{n}+d_{n}+\ldots+d_{1}
$$

$\alpha_{i}^{n}, \beta_{i}^{j}$ are the scaling and wavelet coefficients, $\varphi^{n}(\mathbf{t})$ is the scaling function at level $n$ and $\psi^{j}(\mathbf{t})$ the wavelet function at level $j ; n$ is the decomposition level; $a_{n}$ is the approximation signal at level $n$ and $d_{j}$ the detail signal at level $j[7,13]$.

This decomposition can be carried out with commercial software packages easily available, a fact increasing the industrial applicability of the diagnosis approach. In this work, the MATLAB Wavelet Toolbox was used.

For the DWT application two main parameters must be selected:

- Type of mother wavelet (and, depending on the case, order of the mother wavelet).

- Number of decomposition levels.

In Section 7, a deeper analysis on the guidelines for the selection of DWT parameters is presented.

\section{Qualitative recognition of the fault-related patterns in the wavelet signals.}

Once the DWT-based method has been applied, the aim is to identify possible patterns appearing in the wavelet signals. The recognition of a characteristic pattern associated with a particular fault enables the diagnosis of the presence of that fault in the machine.

An important advantage lies in the fact that, whereas in the classical approach (FFT spectrum of the steady-state current) other phenomena might introduce frequency components masking completely the fault-related frequencies, in the case of the DWT approach, if these phenomena are present, they are circumscribed to certain frequency bands. Therefore it is always possible to detect, at least partially, the evolution of fault-related components. This is due to the time-frequency nature of the decomposition, which provides much more information than the single frequency peaks appearing the FFT spectrum.

In Section 5, experimental results with real machines are presented. There, the patterns caused by each fault in the wavelet signals are accurately described.

A further step, out of the purpose of this paper, would consist of the automation of the pattern-recognition process. This could be achieved with modern artificial intelligence techniques such as neural networks or fuzzy logic. They would enable the further implementation of the fault-diagnosis algorithm in portable condition monitoring devices.

\section{Computation of quantification parameters}

The qualitative recognition of characteristic patterns created by each fault only enables to confirm the presence of the corresponding fault in the machine. However, at this stage, the level of failure is unknown; the fault might be just in an incipient stage or it might be quite advanced in the machine. For instance, in the case of broken rotor bars, they could consist of an incipient breakage, a complete bar breakage, two broken bars, three broken bars...In the case of mixed eccentricities, the degree of failure could be also quite different (10\% eccentricity, $20 \%, 30 \% \ldots$..). Therefore, it becomes necessary to provide indicators for quantifying the degree of failure in the machine.

As it will be seen in Section 5, the amplitudes of the oscillations caused by each fault in the wavelet signals increase when the severity of the fault does. Therefore, it is possible to build the quantification parameters on the basis of the amplitude (or the energy) of the wavelet signals affected by the fault.

This quantitative approach, based on the introduction of quantification parameters, complements the qualitative perspective based on the detection of the patterns; first, with the pattern recognition, the fault is identified and, later, with the quantification parameters the degree of failure is determined.

In Section 6, parameters for quantifying both studied faults are defined and computed for each machine.

\section{Experimental results}

In this section the presented methodology is applied to the diagnosis of several machines under different fault and operation conditions. The tests were performed in the laboratory, using commercial cage motors with 4 
poles, 28 rotor bars, rated $1.1 \mathrm{~kW}, 400 \mathrm{~V}, 50 \mathrm{~Hz}$, coupled to two different DC machines acting as loads (load 1 (direct coupling) and load 2 (coupling through straps)). Figure 6(a) shows the motor under test.

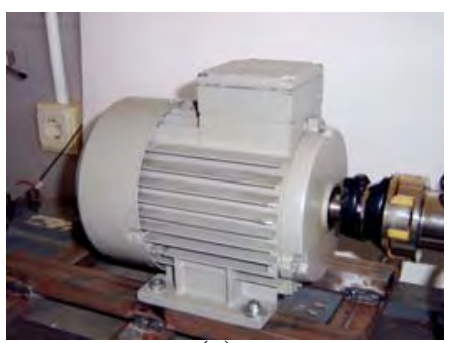

(a)

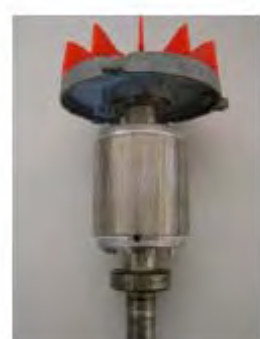

(D)
Fig. 6. (a) $1.1 \mathrm{~kW}$ motor under test (b) Rotor with one broken bar.

A phase current was used as diagnostic signal; this current was captured using a 15/5, class 0.5 current transformer and a $1 \mathrm{~A}, 60 \mathrm{mV}$ shunt; the resulting voltage signal was captured by means a digital oscilloscope with a sampling frequency $f_{s}=5000$ samples/s, and finally transferred to a PC for the analyses. The standard MATLAB Wavelet Toolbox was used for performing the DWT of the signals; Daubechies-44 was selected as mother wavelet. Figures in the next sections show the wavelet signals resulting from the transform, as well as their associated frequency bands.

\subsection{Unloaded healthy machine}

Figure 7 shows the DWT of the startup current for the healthy motor coupled to load 1 . The wavelet signals resulting from the analysis (approximation and detail signals) do not show any significant oscillations once the electromagnetic transient, occurring at the beginning of the startup in every machine, is finished. This indicates the absence of any fault component, confirming the healthy condition of the machine.

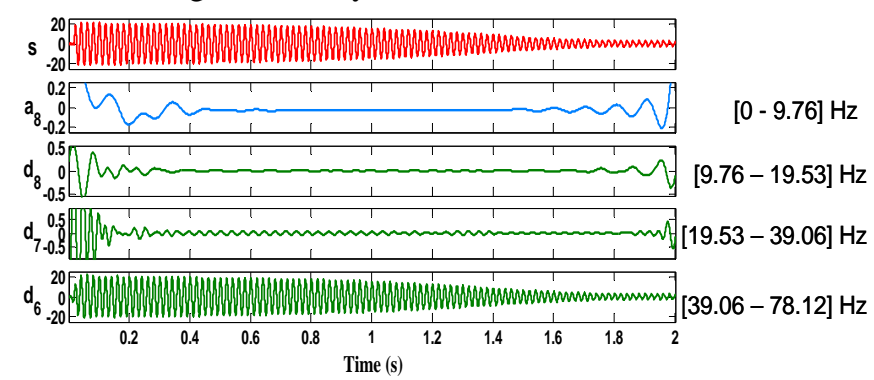

Fig. 7. 8-level DWT of the startup current for the unloaded healthy machine.

\subsection{Unloaded machine with one broken bar}

A bar breakage was artificially forced in the laboratory, by drilling a hole in the selected rotor bar. Figure 6 (b) shows the rotor after the breakage. Figure 8 shows the application of the DWT for the case of a machine with 1 broken bar and coupled to load 1. Clear oscillations appear in the wavelet signals resulting from the analysis. Moreover, they are arranged in such a way that they reflect the evolution in frequency of the left sideband component created by the breakage (first decreasing from the supply frequency $f$ towards $0 \mathrm{~Hz}$ and later increasing towards the supply frequency again).

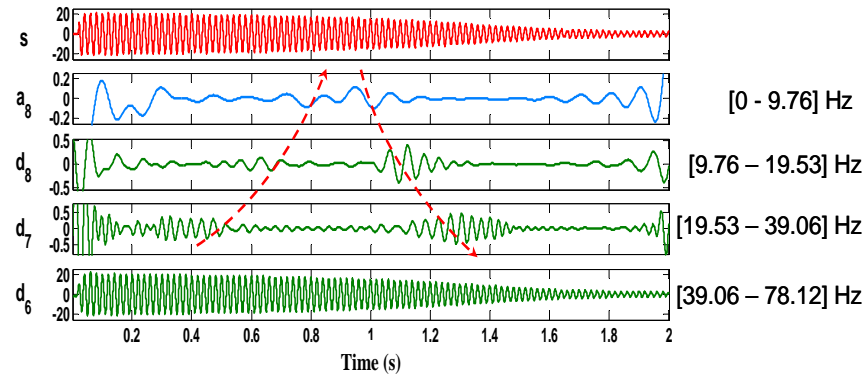

Fig. 8. 8-level DWT of the startup current for the unloaded machine with one broken bar.

If the classical diagnosis methodology, based on the FFT of the steady-state current, was applied in this case the diagnosis conclusion would not be reached. This is due to the fact that the machine is unloaded and, therefore, the slip $s$ is very low, so the sideband components given by (1) overlap the supply frequency $f$. This is shown in Figure 9, where the sidebands are not detectable due to this fact.

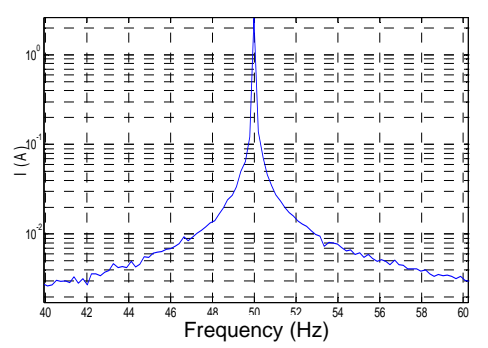

Fig. 9. FFT of the steady state current for the unloaded machine with one broken bar. 


\subsection{Unloaded machine with two broken bars}

Figure 10 shows the application of the diagnosis methodology to an unloaded machine with two broken bars and coupled to load 1 . The conclusion is similar to that of the previous case; the characteristic pattern caused by the evolution of the left sideband is clear in the wavelet signals resulting from the DWT. Moreover, the oscillations within the signals $a_{8}, d_{8}$ and $d_{7}$ have higher amplitudes, due to the higher degree of severity of the fault, in comparison with that of the previous case. This suggests the possibility of introducing parameters for quantifying the degree of severity of the fault based on the energy of the wavelet signals.

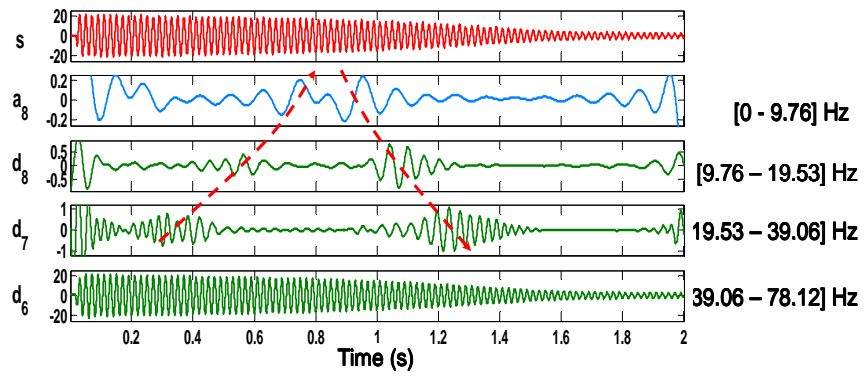

Fig. 10. 8-level DWT of the startup current for the unloaded machine with two broken bars.

\subsection{Unloaded machine started through soft- starter}

This test was carried out using the unloaded machine with one broken bar, coupled to load 2 and started by means of a soft starter. The soft starter controls the voltage supplied to the motor during the startup, increasing it progressively during the transient. This starting method is also common in the industrial environment. Figure 11 shows schematically the testbed for the experiment. Figure 12 shows the DWT analysis of the startup current for this case. The characteristic pattern caused by the evolution of the left sideband appears clearly, confirming also the validity of the approach in this situation.

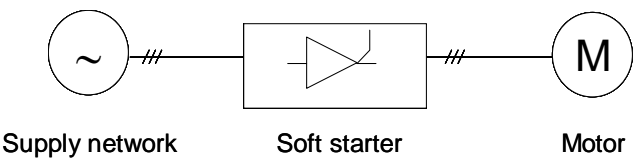

Fig. 11. Simplified scheme for the test.

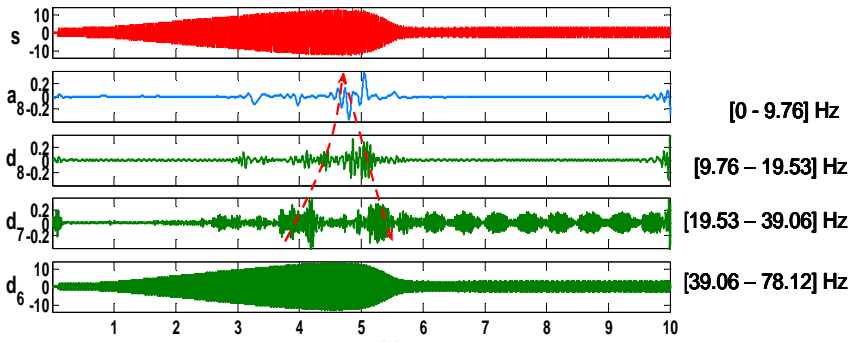

Fig. 12. 8-level DWT of Tipe (startup current for the unloaded machine with one broken bar started through soft-starter.

\subsection{Machine with mixed eccentricity}

Figure 13 shows the application of the methodology for a machine with mixed eccentricity, considering now 6 decomposition levels. The evolution of the aforementioned fault components is clearly noticed; there is one component whose frequency evolves from $50 \mathrm{~Hz}$ to $25 \mathrm{~Hz}$ during the transient and a second one evolving from $50 \mathrm{~Hz}$ to $75 \mathrm{~Hz}$. Therefore, a characteristic pattern different from that associated with the bar breakage arises.

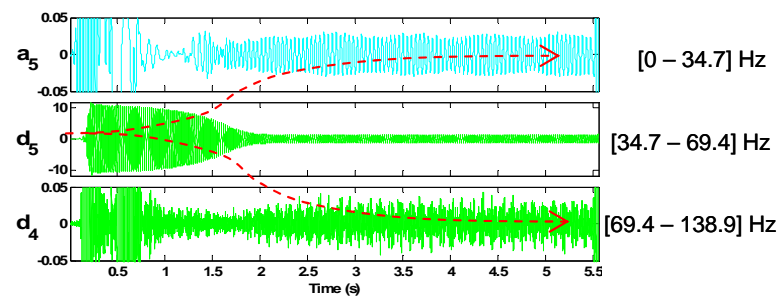

Fig. 13. 6-level DWT of the startup current for the machine with mixed eccentricity.

\section{Introduction of quantification parameters}

Once the condition of the machine has been preliminarily diagnosed, using the qualitative identification of characteristic patterns, it is necessary to compute the quantification parameter defined for the corresponding fault, in order to quantify the degree of failure in the machine.

In the case of rotor asymmetries, a quantification parameter $\gamma_{a s y m}$ was defined in previous works [16]. It was based on the energy of the wavelet signal with the next level higher than the signal containing the fundamental. This parameter is given by (8).

$$
\gamma_{\text {asym }}(d B)=10 \cdot \log \left[\frac{\sum_{j=N b}^{N s} i_{s t j}{ }^{2}}{\sum_{j=N b}^{N s}\left[d_{n f+1}(j)\right]^{2}}\right]
$$




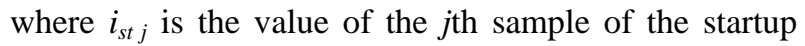
current signal $i_{s t}(\mathrm{t}) ; d_{n f+1}(j)$ is the $j$ element of the detail with level $n f+1$ ( $n f=$ level of the signal containing the fundamental); $N_{s}$ is the number of samples of the signal, until reaching the steady-state and $N_{b}$ is the number of samples between the origin of the signals and the extinction of the oscillations due to border effect.

Since the quantification parameter $\gamma_{a s y m}$ is the ratio between the energy of the original startup current signal and the energy of the detail signal used for diagnosis purposes and due to the fact that this detail signal increases its energy when the level of failure is greater, it is logical that the value of the fault parameter decreases as the level of failure increases. The reason for the increment in energy of the detail signal per unit energy of the total current signal lies on the fact that this signal contains the partial transient evolution of the left sideband component during the startup. Since when the machine is healthy this sideband is not present, the energy of this detail signal is low which implies a high value of the quantification parameter. On the other hand a faulty machine implies a high energy for the detail signal and, therefore, a low value of the parameter.

According to the experience achieved by tests carried out in motors with this range of powers (few $\mathrm{kW}$ ), a value for $\gamma_{\text {asym }}$ higher than $40 \mathrm{~dB}$ is indicative of a healthy condition in the machine. Values between $30 \mathrm{~dB}$ and $40 \mathrm{~dB}$ mean that a partial breakage or one broken bar is present in the machine. Values around $30 \mathrm{~dB}$ or lower are usually obtained when at least two bars are broken.

Table I shows the results obtained when computing this indicator for different cases tested, as well as the deviations with respect the healthy condition for each machine. The values obtained confirm the ranges commented above.

In the case of mixed eccentricities, a quantification parameter $\gamma_{\text {mecc }}$ could be also defined, based on the energy of the approximation signal with the next level higher than that containing the fundamental component. This parameter would be according to (9).

$$
\gamma_{\text {mecc }}(d B)=10 \cdot \log \left[\frac{\sum_{j=N b}^{N s} i_{\text {st } j}^{2}}{\sum_{j=N b}^{N s}\left[a_{n}(j)\right]^{2}}\right]
$$

where $i_{s t j}$ is the value of the $j$ sample of the current signal; $a_{n}(j)$ is the $j$ element of the order $n$ approximation signal; $N_{s}$ is the number of samples of the signal, after finishing the first 10 cycles in the steady-state regime and $N_{b}$ is the number of samples between the origin of the signals and the extinction of the oscillations due to border effect.

Table 1. Computation of the indicator $\gamma_{\text {asym }}$ for each case tested and deviation with respect the healthy condition $\left(\Delta \gamma_{\text {asym }}\right)$.

\begin{tabular}{|c|c|c|c|}
\hline Machine & Condition & $\gamma_{a s v m}$ & $\Delta \gamma_{a s y m}$ \\
\hline $\begin{array}{c}1.1 \mathrm{~kW} \text { motor } \\
\text { coupled to load } 1\end{array}$ & Healthy & 47.1 & - \\
\hline $\begin{array}{l}1.1 \mathrm{~kW} \text { motor } \\
\text { coupled to load } 1\end{array}$ & 1 broken bar, unloaded & 37 & -10.1 \\
\hline $\begin{array}{l}1.1 \mathrm{~kW} \text { motor } \\
\text { coupled to load } 1\end{array}$ & 1 broken bar, $80 \%$ load & 36.2 & -10.9 \\
\hline $\begin{array}{l}1.1 \mathrm{~kW} \text { motor } \\
\text { coupled to load } 1\end{array}$ & 1 broken bar, full-load & 35.2 & -11.9 \\
\hline $\begin{array}{l}1.1 \mathrm{~kW} \text { motor } \\
\text { coupled to load } 1\end{array}$ & $\begin{array}{c}2 \text { broken bars, } \\
\text { unloaded }\end{array}$ & 30.6 & -16.5 \\
\hline $\begin{array}{l}1.1 \mathrm{~kW} \text { motor } \\
\text { coupled to load } 1\end{array}$ & $\begin{array}{c}2 \text { broken bars, } 60 \% \\
\text { load }\end{array}$ & 30.0 & -17.1 \\
\hline $\begin{array}{l}1.1 \mathrm{~kW} \text { motor } \\
\text { coupled to load } 1\end{array}$ & 2 broken bars, full load & 30.1 & -17 \\
\hline $\begin{array}{l}1.1 \mathrm{~kW} \text { motor } \\
\text { coupled to load } 2\end{array}$ & Healthy & 44.4 & - \\
\hline $\begin{array}{l}1.1 \mathrm{~kW} \text { motor } \\
\text { coupled to load } 2\end{array}$ & 1 broken bar, unloaded & 35.6 & -8.8 \\
\hline $\begin{array}{l}1.1 \mathrm{~kW} \text { motor } \\
\text { coupled to load } 2\end{array}$ & 1 broken bar, $80 \%$ load & 35.4 & -9 \\
\hline $\begin{array}{l}1.1 \mathrm{~kW} \text { motor } \\
\text { coupled to load } 2\end{array}$ & 1 broken bar, full-load & 35.1 & -9.3 \\
\hline $\begin{array}{l}1.1 \mathrm{~kW} \text { motor } \\
\text { coupled to load } 2\end{array}$ & $\begin{array}{l}2 \text { broken bars, } \\
\text { unloaded }\end{array}$ & 30.7 & -13.7 \\
\hline $\begin{array}{c}1.1 \mathrm{~kW} \text { motor } \\
\text { coupled to load } 2\end{array}$ & 2 broken bars, full load & 31.8 & -12.6 \\
\hline
\end{tabular}

\section{Additional considerations for the application of the method}

The different experiments performed showed the suitability of the method for the diagnosis of electromechanical faults introducing slip-dependant components. Nevertheless, additional considerations need to be done regarding the different parameters of the DWT decomposition, such as the type of mother wavelet, the order of the mother wavelet or the number of decomposition levels.

With regards to the type of mother wavelet, the Daubechies family was well suited for the application of this method, due to its inherent properties (this family has well-known mathematical properties and it is 
included in many commercial software packages, thus not requiring any special algorithm or custom design for its application), although other families (symlet, biorthogonal, Gaussian, and specially dmeyer) also enable a clear detection of the patterns, despite their different mathematical characteristics. As an example, Figures 14 (a) and (b) show the application of the method for the case of unloaded machine with one broken bar and coupled to load 2, using symlet-30 and dmeyer, respectively. The similarities between both figures are obvious, appearing the characteristic pattern caused by the sideband.

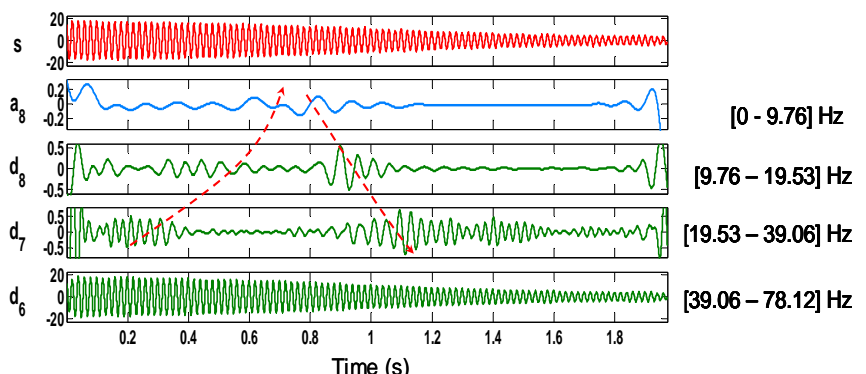

(a)

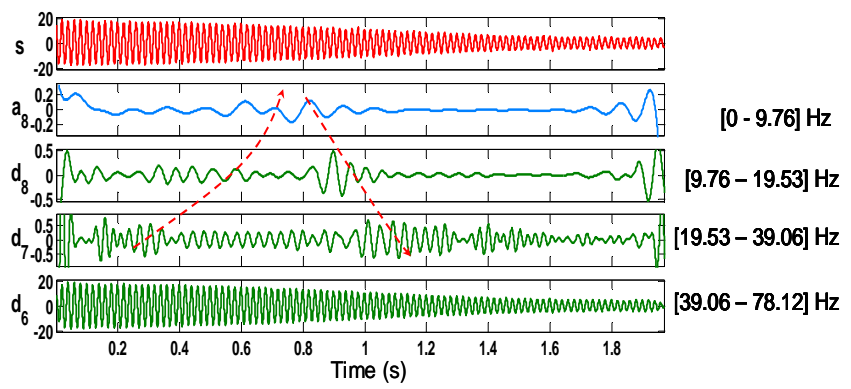

(b)

Fig. 14. 8-level DWT of the startup current for the unloaded machine with one broken bar using: (a) symlet-30, (b) dmeyer.

When using the Daubechies family, an important fact observed was the overlapping between the frequency bands associated with successive wavelet signals resulting from the DWT of the current. This is due to the fact that the wavelet signals act as non-ideal filters, extracting the components of the signal included within a certain frequency band that can overlap partially with the adjacent band $[7,15]$. In this sense, it was observed that, when using a high-order Daubechies wavelet for signal decomposition, the overlap was smaller than when using a low-order one. In other words, high-order wavelets behave as more ideal filters, a fact that helps to avoid partially the overlap between frequency bands.

Finally, the number of decomposition levels $\left(n_{d}\right)$ is related to the sampling frequency of the signal being analysed $\left(f_{s}\right)$. For the diagnosis of broken rotor bars, this parameter has to be chosen in such a way that the DWT supplies at least three high-level signals (two details and an approximation) with frequency bands below the supply frequency $f$; this condition implies:

$$
n_{d} \geq n_{f}+2
$$

being $n_{f}$ the level of the detail which contains the supply frequency, that can be calculated using (11).

$$
2^{-\left(n_{d}+1\right)} \cdot f_{s}<f
$$

This condition means that the lower limit of the frequency band of the $n_{f}$ level detail is lower than the supply frequency.

Thus:

$$
n_{d}>\frac{\log \left(f_{s} / f\right)}{\log (2)}+1 \quad \text { (integer) }
$$

Therefore, for the previous examples, the condition implies:

$$
n_{d}>\frac{\log (5000 / 50)}{\log (2)}+1=7.6438
$$

Which leads to $n_{d}=8$, which is the number of levels selected for the diagnosis of this fault.

With regards the diagnosis of the mixed eccentricity, similar considerations lead to the following expression for the selection of the optimum number of levels:

$$
n_{d}=\operatorname{int}\left(\frac{\log \left(f_{s} / f\right)}{\log (2)}\right)
$$

In our case, this leads to $n_{d}=6$, which was the number of levels selected (see Fig. 13).

\section{Conclusions}

A diagnosis methodology is presented in this paper to diagnose the presence of electromechanical faults in electrical machines. It is based on the application of the DWT to the stator startup current and the further recognition of characteristic patterns created by each fault in the wavelet signals. 
Several faulty cases are presented in the paper, all them confirming the validity of the approach, even in some cases in which the classical FFT methodology, currently used in the industrial environment, might not lead to correct results.

The method admits the quantification of the degree of failure using parameters based on the energy of the wavelet signals resulting from the analysis.

Further work would be based on the application of image recognition algorithms for the automatic identification of these characteristic patterns, which could be the basis for the implementation of portable diagnosis devices.

\section{Acknowledgements}

The research leading to these results has received funding from the European Community's Seventh Framework Programme FP7/2007-2013 under Grant Agreement n 224233 (Research Project PRODI "Power plant Robustification based on fault Detection and Isolation algorithms"). The authors also thank 'Vicerrectorado de Investigación, Desarrollo e Innovación of Universidad Politécnica de Valencia' for financing a part of this research through the program 'Programa de Apoyo a la Investigación y Desarrollo (PAID-06-07).

\section{References}

1. W.T. Thomson, M. Fenger, Current signature analysis to detect induction motor faults, IEEE Industry Applications Magazine, July/August 2001, pp. 26-34.

2. J.C. Augusto, J. Liu, P. McCullagh, H. Wang, J-B. Yang, Management of Uncertainty and Spatio-Temporal Aspects for Monitoring and Diagnosis in a Smart Home, International Journal of Computational Intelligence Systems, Vol.1-4, December 2008, pp.361-378.

3. M. H. Benbouzid, A review of induction motors signature analysis as a medium for faults detection, IEEE Transactions on Industrial Electronics, Vol. 47, No. 5, October 2000.

4. G.B Kliman, J Stein, and R.D. Endicott, Noninvasive Detection of Broken Rotor Bars in Operating Induction Motors, IEEE Transactions on Energy Conversion, Vol. 3, No. 4, pp. 873-879, December 1988.

5. J.R Cameron, W.T. Thomson and A.B. Dow, Vibration and current monitoring for detecting airgap eccentricity in large induction motors, IEE Proceedings, Vol. 133, pt. B, No. 3, May 1986, pp. 155-163.

6. R.R. Schoen and T.G. Habetler. Evaluation and Implementation of a System to Eliminate Arbitrary Load Effects in Current-Based Monitoring of Induction
Machines, IEEE Transactions on Industry Applications, Vol. 33, No. 6, November/Dec.1997, pp. 1571-1577.

7. J.A. Antonino-Daviu, M.Riera-Guasp, J.Roger-Folch and M.P. Molina, Validation of a New Method for the Diagnosis of Rotor Bar Failures via Wavelet Transform in Industrial Induction Machines, IEEE Transactions on Industry Applications, Vol. 42, No. 4, July/August 2006, pp.990-996.

8. M. Riera-Guasp, J. Antonino-Daviu, J. Roger-Folch and M.P. Molina, The Use of the Wavelet Approximation Signal as a Tool for the Diagnosis and Quantification of Rotor Bar Failures, IEEE Transactions on Industry Applications Vol. 44, No. 3, May-June 2008, pp. 716726.

9. J. Antonino-Daviu, P. Jover, M. Riera-Guasp, J. RogerFolch and A. Arkkio, DWT Analysis of Numerical and Experimental Data for the Diagnosis of Dynamic Eccentricities in Induction Motors, Mechanical Systems and Signal Processing, Elsevier, Vol. 21, No. 6, August 2007, pp. 2575-2589.

10. H. Douglas, P. Pillay and A. Ziarani , Broken rotor bar detection in induction machines with transient operating speeds, IEEE Transactions on Energy Conversion, Vol. 20, No.1, March 2005, pp.135-141.

11. Z. Zhang and Z. Ren, A novel detection method of motor broken rotor bars based on wavelet ridge, IEEE Transactions on Energy Conversion, Vol. 18, No. 3, September 2003, pp. 417-423.

12. J. Antonino-Daviu, M. Riera-Guasp, J. Roger-Folch, F. Martínez-Giménez, A. Peris, Application and Optimization of the Discrete Wavelet Transform for the Detection of Broken Rotor Bars in Induction Machines, Applied and Computational Harmonic Analysis, Elsevier, Vol. 21, September 2006, pp. 268-279.

13. C.S. Burrus, R.A. Gopinath and H. Guo, Introduction to Wavelets and Wavelet Transforms. A primer, Prentice Hall, 1998.

14. R.Polikar, The wavelet tutorial http://engineering.rowan.edu/ polikar/WAVELETS/WTt utorial.html

15. T. Tarasiuk, Hybrid wavelet-Fourier Spectrum Analysis, IEEE Transactions on Power Delivery, Vol. 19, No. 3, pp. 957-964, July 2004.

16. M. Riera, J. A. Antonino, J. Roger-Folch, and M.P. Molina, Definition of DWT-based parameters for the quantification of rotor bar breakages in industrial induction machines, Proc., ISEF 2005, Baiona, Spain, September 17-19, 2005. 